Biomedical Research Centre, did his undergraduate, graduate and postdoctoral work in physics before switching to bioinformatics and next-generation sequencing; and McVean sees many recruits enter the field from banking and finance.

Statistician Yun Li joined the faculty of the University of North Carolina in Chapel Hill after earning her doctoral degree in biostatistics at the University of Michigan in 2009. In her undergraduate degree, Li had minored in computer science; she then earned a master's in statistics before starting her doctorate. While working on her $\mathrm{PhD}$, Li developed data-analysis methods for the 1000 Genomes Project, a multinational study in which more than 1,000 individuals' genomes are being sequenced. She says that the hands-on experience working with what she calls "dirty" data - raw data whose characteristics and limitations have not been fully explored by researchers - has been invaluable in her current position.

"A typical genetic study nowadays will need to analyse millions or tens of millions of variants in

$\supset$ tens of thousands of

오․ individuals," says Li,

' who is now developing ways to work with large data sets and applying these and other methods to disease-focused studies. "This entails skills both to identify problems - which is important because many issues are typically not defined for data from cutting-edge research - and to solve problems."

Whether trainees are interested in an academic or indus-

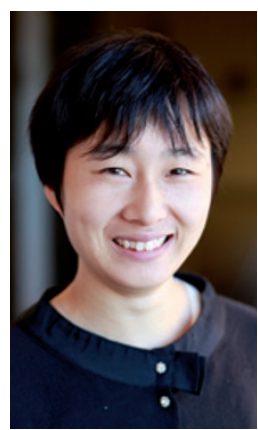

"A typical genetic study nowadays will need to analyse millions of variants." Yun Li trial job, it is computer-science skills that will help them to secure it. By far the most successful candidates are those who can not only write software, but also work with distributed computing systems, and computer operating systems such as Linux and Unix, say those in the field. "The more you understand software and computer science, the better off you are; writing software is $90 \%$ of what we're doing," says Alexander.

For a field that is likely to continue its rapid change, the only sure thing is that data sets will continue to get bigger, and those who know how to handle them will be in high demand..

Erika Check Hayden reports for Nature from San Francisco.

COLUMN

\title{
Friend or foe?
}

\section{It is difficult to balance the benefits of collaboration and competition, argues Lydia Murray.}

A s a PhD student, learning to navigate the murky waters of collaboration and competition is pretty confusing. I recently attended my first conference and never mind the name badges, I wanted to tattoo 'FRIEND' or 'FOE' on people's foreheads. Given that a researcher's publications are often months, if not years, behind their current lab work, it is hard to discover who is working on what. Knowing when to share unpublished ideas and when to practise your poker face can be a nightmare for an earlycareer scientist.

Why is it so hard? One reason is that science is a truly integrated discipline: completely independent fields are rare. As multiple groups generate data around the world, hypotheses evolve, and the direction of a scientist's research can change. One group's work might bleed into another's field of interest. So when two labs find their investigations becoming a bit too close for comfort, how do they decide whether to collaborate or compete?

Collaborations can be brilliant. Bringing together different skills and expertise offers fresh insight into old challenges and opens up new avenues of research. However, sharing a research theme does not always result in happy scientist families. Competition can overshadow the collaborative spirit and hinder progress.

Of course, competition is essential to science. It can stimulate motivation and productivity for labs addressing the same questions with conflicting hypotheses: the opportunity to deliver a scientific 'I told you so' is an appealing incentive. Healthy rivalry keeps fields exciting and ensures that all angles of research questions are considered.

However, when different groups are testing the same hypothesis, the contest is often simply a race to publication. The group that wins increases its citation number and strengthens its reputation. But does this justify the duplicated data, man hours and, potentially, taxpayers' money? In the current economic climate, I find it hard to understand how this style of competition remains prevalent.

There is at least one intermediate path between collaboration and competition: labs can coordinate publications. Instead of rushing through projects in parallel, they can agree to submit simultaneously and address a complementary range of questions. Without the time

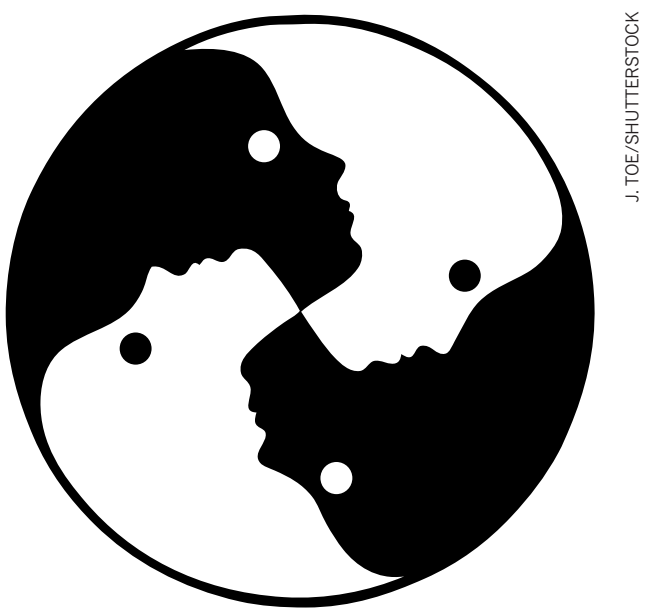

pressure, compromises in research quality are reduced. Ultimately, the journal audience can read a far more comprehensive story.

But many labs continue to jealously guard their progress and sacrifice paper quality for personal recognition. Should such egotism be acceptable in science, the main aims of which are, ideally, discovery and innovation, rather than accolades for its practitioners? As a young researcher, I am puzzled that a community reliant on integrity and transparency is tolerant of lies and misdirection in the publications race.

That said, I'm not sure it would be prudent to advise young scientists always to speak freely at conferences and discard the poker face. Unless every person in the room does the same thing, you will eventually get scooped. As physicist Max Planck once wrote, "A new scientific truth does not triumph by convincing its opponents and making them see the light, but rather because its opponents eventually die, and a new generation grows up that is familiar with it”. Young scientists will have a crucial role in establishing a culture of greater cooperation amid a global scientific enterprise increasingly populated with far-flung collaborations. But we also need to recognize the importance of a bit of competition - and the reality that researchers will probably always be on the lookout for both friend and foe.

Lydia Murray is a PhD student in the department of medicine, veterinary and life sciences at the University of Glasgow, UK. 